\title{
Research Paper: Clinical Significance of Diffusion-weighted Magnetic Resonance Imaging on Treatment Efficacy in MS Patients With Acute Attacks
}

\author{
Faezeh Asaadi $^{1}$ (D), Fariborz Faeghi ${ }^{*}$ (D), Farzad Ashrafi² ${ }^{(D)}$, Morteza Sanei Taheri $^{3}$ (D)
}

1. Department of Radiology Technology, School of Allied Medical Sciences, Shahid Beheshti University of Medical Sciences, Tehran, Iran

2. Neurology Brain Mapping Research Center, Shahid Beheshti University of Medical Sciences, Tehran, Iran.

3. Department of Radiology, Shohadaye Tajrish Hospital, Tehran, Iran.

\begin{tabular}{|c|c|}
\hline $\begin{array}{l}\text { Use your device to scan } \\
\text { and read the article online }\end{array}$ & Citation Asaadi, F., Faeghi, F., Ashrafi, F., \& Sanei Taheri, M. (2021). Clinical Significance of Diffusion-weighted Magnetic \\
\hline 口ifiritar & $\begin{array}{l}\text { Resonance Imaging on Treatment Efficacy in MS Patients With Acute Attacks. Basic and Clinical Neuroscience, 12(6), } 729 \text { - } \\
\text { 736. http://dx.doi.org/10.32598/bcn.2021.1560.1 }\end{array}$ \\
\hline 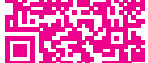 & doi'http://dx.doi.org/10.32598/bcn.2021.1560.1 \\
\hline
\end{tabular}

(c) (1) (3)

Article info:

Received: 20 Oct 2018

First Revision: 14 May 2019

Accepted: 04 Jan 2020

Available Online: 01 Nov 2021

Keywords:

Multiple Sclerosis, Diffusion Magnetic Resonance Imaging, Demyelinating diseases, Methylprednisolone

\section{A B S T RAC T}

Introduction: Despite various imaging methods, the accurate diagnosis of numerous neurodegenerative diseases remains controversial. Using advanced imaging techniques, like diffusion-weighted imaging, can help the early detection of Multiple Sclerosis (MS) and evaluation of the treatment efficacy in these patients.

Methods: In total, 24 MS patients with acute attack and 30 healthy subjects were considered in our study. Region of Interest (ROI) was defined for acute and chronic plaques and NormalAppearing White Matter (NAWM) in the patients' group. In the normal group, ROI only was mapped in the white matter in the same regions of the patient. All MS patients were receiving Methylprednisolone for 3 to 5 days. The rate of clinical disability in these patients was also evaluated based on the Expanded Disability Status Scale (EDSS) index. Finally evaluate changes of ADC values of plaques and NAWM before and after treatment.

Results: The Apparent Diffusion Coefficient (ADC) values of acute plaques, the ADC values of NAWM, the number of enhancement in T1w, and EDSS values suggested a significant difference after treatment compared to before treatment. However, the ADC values of chronic plaques revealed no significant difference after treatment. There was a significant positive correlation between the difference in EDSS values before and after treatment.

Conclusion: The study results demonstrated that using diffusion technique and ADC values analysis is a proper non-invasive method for MS diagnosis and evaluating treatment efficacy in these patients.

\section{* Corresponding Author:}

Fariborz Faeghi, PhD.

Address: Department of Radiology Technology, School of Allied Medical Sciences, Shahid Beheshti University of Medical Sciences, Tehran, Iran. Tel: +98 (912) 7171638

E-mail:ffaeghi@sbmu.ac.ir 


\section{Highlights}

- The obtained results suggested that the mean $\mathrm{ADC}$ for acute plaques and normal white matter significantly decreased after methylprednisolone treatment.

- Our study indicated a strong correlation between variations in EDSS, the mean ADC for acute plaques, and normal white matter.

- The collected results indicated that the number of enhanced plaques decreased after treatment. Besides, there was a positive correlation between its variations and EDSS.

\section{Plain Language Summary}

Multiple Sclerosis (MS) is a common inflammatory disorder of the central nervous system that could result in physical and mental disabilities in patients. Disease progression usually manifests as a series of attacks. Although there is no proven cure for MS, different treatment strategies aim to modify the cause of the disease, manage its symptoms, and prevent and postpone disability. The most common therapy in acute attacks is using corticosteroid drugs. In addition to the treatment, evaluating the success rate of treatment was also challenging. Historically, clinical assessments method (e.g. EDSS) have been used as the baseline for measuring the therapy's efficiency. Several supplementary methods, including imaging techniques, are introduced to address this issue. Conventional MRI imaging with injection has been widely accepted to assess the treatment. However, because of the modest sensitivity of conventional MRI to detect subtle pathological changes, there is a poor correlation between its findings and patients' disability. This study moved from conventional MRI to advanced techniques, such as DWI and its quantitative index named ADC value. This technique can provide information about microstructural changes in MS patients. This method does not require injection, so there are no probable adverse effects and lower scan time. This study emphasizes changes in ADC value and EDSS before and after treatment with methylprednisolone. Our results suggested s that ADC values and EDSS after treatment are significantly different from their typical values. ADC values can be used as a biomarker to evaluate treatment efficiency, yet it is not objective enough to use it alone. So, the combination of DWI imaging with conventional methods might be beneficial in assessing treatment efficiency in MS patients.

\section{Introduction}

M

ultiple Sclerosis (MS) is among the most common inflammatory autoimmune neurological disorders of the Central Nervous System (CNS), leading to disability in young adults (Davoudi, Foroughipour Torabi, Layegh, Matin, \& Shoeibi, 2016; Simon, 1997). MS typically presents in adults aged 20 to 45 years (Goldenberg, 2012). The cause of the onset of MS remains unknown. Still, the combination of genetic and non-genetic factors, such as the virus, environmental factors, etc., seems to lead to an autoimmune disease. From the pathophysiological viewpoint in this disease, macrophages introduce myelin antigens to $\mathrm{T}$ cells and cause the proliferation of $\mathrm{T}$ cells. They crossed the blood-brain barrier by intercellular adhesion molecules and entered the central nervous system. These cells then release the cytokines and cause an immune response. In some parts of the world, the prevalence of MS is higher.
Iran seems to be among the areas with high MS prevalence (Etemadifar et al., 2013).

There is no specific clinical test for MS diagnosis. Diagnosis is based on symptoms of illness, lumbar punctures for Cerebrospinal Fluid (CSF) analysis, and Magnetic Resonance Imaging (MRI) (Ghasemi, Razavi, \& Nikzad, 2017). MRI is a non-invasive and high-sensitivity method for MS diagnosis. Conventional MRI techniques lack proper sensitivity to display minor pathologic variations in the patients. Due to these limitations, using quantitative techniques, like Diffusion-Weighted Imaging (DWI), is developed to diagnose neurodegenerative diseases (Hannoun et al., 2015). DWI is a noninvasive technique, i.e., sensitive to the random motion of water molecules in tissue.

In the brain, white matter, due to the myelin sheath, i.e., like a barrier against water release, is anisotropic water diffusion along the neural pathway fibers. In neurodegenerative diseases, like MS, where myelin is damaged, an- 
isotropy is reduced, and the myelin barrier is affected. In these patients, valuable information can be obtained about the white matter state by analyzing and processing data from diffusion images (Aung, Mar, \& Benzinger, 2013). Given the few studies to examine the DWI technique as a method for evaluating the response to treatment, this study emphasized the importance of the DWI imaging sequence in assessing the response to treatment and its potential to assess therapeutic interventions in patients with MS.

\section{Methods}

This study examined 24 MS patients with acute attacks (average age of 30 years, 17 women $\& 7$ men). The degree of clinical disability in these patients was assessed based on the EDSS index according to Table 1. The control group consisted of 30 volunteers (mean age: 30.47 ; 18 women \&d 12 men) without a history of neurological disease and normal brain MRI. All MS patients were treated with Methylprednisolone for 3 to 5 days. MRI was obtained from these patients before and after medication.

The study's inclusion criteria included the following: Patients with MS referring to the Academic Neurology Center. The acute attack was diagnosed in these patients per symptoms, clinical history, physical examination, and laboratory tests such as a lumbar puncture.

The exclusion criteria included patients with a history of fever, patients with any other neurological disease, and patients with claustrophobia.

\section{Ethical considerations}

The project stages have been described for all participants in this study, and then the informed consent form has been signed. After the referral to the MRI department, the study subjects underwent examinations, imaging, and testing. Next, they were treated with methylprednisolone for 3 to 5 days under medical supervision. MRI was taken from the brains of the patients before and immediately after treatment.

In this study, MRI was performed by Siemens Avanto 1.5 Tesla. The applied protocols include: T2 weighted FSE (TR: 5600, TE: 117), Flair (TR: 7000, TE: 84, TI: 2216.7), and images before and after injection T1 weighted SE (TR: 550, TE: 10). The diffusion imaging sequence was performed before injection with the following parameters: performed Single-shot EPI (TR: 5000, TE: 102, NEX: 3, slice thickness: $5 \mathrm{~mm}$ with no gap and two b values $\left(0,1000 \mathrm{~s} / \mathrm{mm}^{2}\right)$.
An expert radiologist evaluated all images. Plaques have been detected on $\mathrm{T}_{2} \mathrm{w}$ and FLAIR images. In addition, it has been assessed at post-contrast $\mathrm{T}_{1} \mathrm{~W}$ images taken 10 minutes after Gd injection. Diffuse or ring enhancement plaques were considered acute plaques. Evaluation of the number and intensity plaques in postcontrast images before and after treatment was based on visual analysis. The spatial sites of all plaques (both acute and chronic) were determined on Apparent Diffusion Coefficient (ADC) map images. For ADC analysis, both before and after treatment Region of Interest (ROI) were drowned on $b=0$ images and copied and pasted onto ADC mapping images by the RAIDIENT software. The average of all plaque has been calculated if more than one plaque were in the patient's images.

For NAWM in the patient and control group, bilateral ROI was obtained at the frontal and occipital horn of lateral ventricle and centrum semi-oval and paying attention that far away from plaques, ventricular system, and gray matter (Figure 1). Finally, evaluate changes of ADC values of plaques and NAWM before and after treatment. In addition, the correlation between ADC value, contrast enhance changes, and EDSS was evaluated.

Data analysis was performed using SPSS. The Spearman correlation coefficient measured the correlations between the study variables. Continuous variables were expressed as Mean \pm Standard Deviation. T-test and twodimensional tests were used to compare the examined tools. $\mathrm{P}<0.05$ was considered statistically significant.

\section{Results}

In this study, 24 patients with acute MS attacks were in the experimental group, and 30 healthy subjects participated in the control group. The two groups were matched in terms of age. The demographic characteristics of the subjects studied are presented in Table 2.

Based on the obtained results, the mean ADC for acute plaques, ADC for normal white matter, the number of enhanced plaques in $\mathrm{T}_{1} \mathrm{w}$ images after injection, and EDSS were significantly different before and after treatment. However, the mean ADC for chronic plaques was insignificant, although lower after treatment (Table 3).

The mean ADC for acute, chronic, and normal white matter before treatment was significantly different from the mean of these values in the control group. However, the mean $\mathrm{ADC}$ for acute plaques and normal white matter did not differ significantly from the control group after the treatment (Table 4). There was also a relatively strong 
Table 1. Expanded Disability Status Scale (EDSS)

\begin{tabular}{|c|c|}
\hline EDSS Scale & Symptoms \\
\hline 0 & Normal neurological exam \\
\hline $1-1.5$ & No disability \\
\hline $2-2.5$ & Minimal disability \\
\hline $3-3.5$ & Mild- moderate disability \\
\hline $4-4.5$ & Moderate disability \\
\hline $5-5.5$ & Increasing limitation in the ability to walk \\
\hline $6-6.5$ & Walking assistance is needed \\
\hline $7-7.5$ & Confined to wheelchair \\
\hline $8-8.5$ & Confined to bed or chair \\
\hline $9-9.5$ & Completely dependent \\
\hline 10 & Death due to MS \\
\hline
\end{tabular}
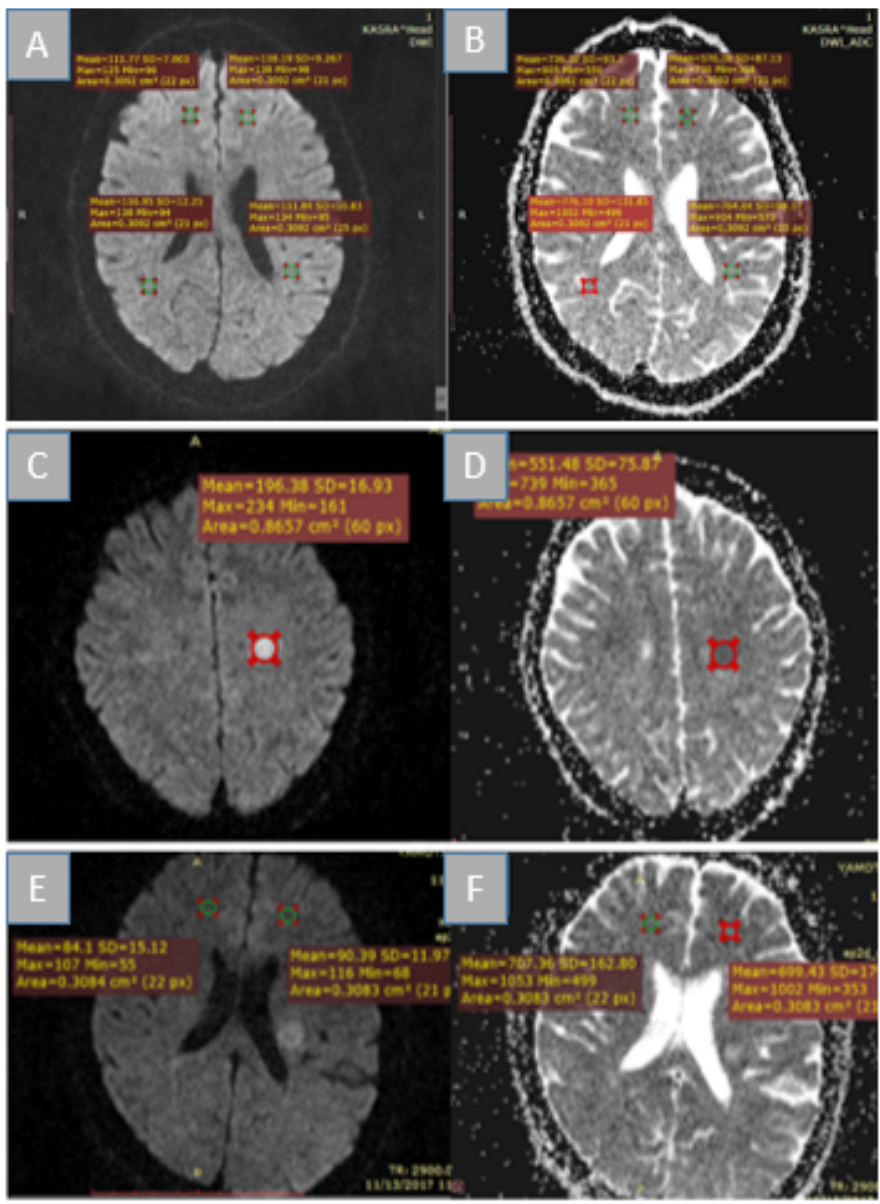

NEUR SCIENCE

Figure 1. The ROIs were drawn on the normal white matter of healthy subjects in diffusion (A) and ADC (B) images In MS patients, ROIs were drawn on MS plaque and NAWM in diffusion $(C, E)$ and $A D C(D, F)$ images. 
Table 2. The demographic characteristics of the study participants

\begin{tabular}{|c|c|c|c|}
\hline \multirow{2}{*}{\multicolumn{2}{|c|}{ Characteristics }} & \multicolumn{2}{|c|}{ No. (\%)/ Mean士SD } \\
\hline & & Control Group & MS Group \\
\hline \multirow{3}{*}{ Gender } & Male & $12(40.0)$ & $17(70.8)$ \\
\hline & Female & $18(60.0)$ & $7(29.2)$ \\
\hline & $\mathrm{P}$ & \multicolumn{2}{|c|}{0.82} \\
\hline \multirow{5}{*}{ Age (y) } & $<30$ & $16(3.53)$ & $16(7.66)$ \\
\hline & $30-40$ & $11(7.36)$ & $4(7.16)$ \\
\hline & $>40$ & $3(0.10)$ & $4(7.16)$ \\
\hline & & $30.47 \pm 6.55$ & $30 \pm 8.05$ \\
\hline & P & \multicolumn{2}{|c|}{0.41} \\
\hline
\end{tabular}

and positive correlation between the EDSS difference, the difference between the ADC for acute plaques and normal white matter, and the number of enhanced plaques, i.e., statistically significant. Moreover, there was a poor and insignificant correlation between the ADC for chronic plaques difference and the difference in EDSS (Table 5).

\section{Discussion}

Conventional MRI has a low sensitivity and specificity in determining lesions, such as inflammation, demyelination, and axonal injury. All of these lesions are seen in high-signal $\mathrm{T}_{2} \mathrm{w}$ and FLAIR images (Almolla, Hassan, Raya, \& Hussein, 2016). Therefore, to overcome the limitations of conventional techniques, it is possible to obtain quantitative information about the ultra-structure of brain tissue using techniques such as diffusion and ADC value.

Our study demonstrated that the average ADC value of acute plaques and NAWM significantly decreased after therapy. Additionally, the ADC value of acute plaques and NAWM significantly differed from the control group before treatment but no significant differences after treatment. However, despite a slight drop, the average values for chronic plaques do not suggest a meaningful change. The average value of ADC chronic plaques before and after the treatment increased compared to the averages from the control group. In both steps, we recorded higher average values than the control group.

Increased ADC values in acute plaques can be explained by the contribution of vasogenic edema and inflammation. However, higher ADC values in chronic plaques are thought to reflect expanded extracellular space due to disruption of the axons by the demyelinating process and edema (Eisele et al., 2012; Yurtsever, I., Hakyemez, Taskapilioglu, Erdogan, Turan, \& Parlak, 2008).

It seems that a drop in the $\mathrm{ADC}$ values of acute plaques can be related to the decreasing edema and inflammation due to therapy. However, suboptimal therapeutic re-

Table 3. Comparing the study patients' characteristics before and after treatment

\begin{tabular}{lcc}
\multicolumn{1}{c}{ Parameter } & Mean Pretest-Posttest Difference & P \\
\hline The ADC values of acute plaque & $-24.39 \times 10^{-6}$ & 0.044 \\
\hline The ADC values of normal appearing white matter & $-19.91 \times 10^{-6}$ & -1.667 \\
\hline The Number of enhancement in $\mathrm{T}_{1} \mathrm{w}$ & -1.71 & 0.024 \\
EDSS values & $-11.61 \times 10^{-6}$ & $<0.001$ \\
\hline The ADC values of chronic plaque & & 0.108 \\
\hline
\end{tabular}


Table 4. Comparing ADC values before and after treatment

\begin{tabular}{|c|c|c|}
\hline Variables & Mean Difference With Control Group & $\mathbf{P}$ \\
\hline The ADC values of acute plaque before treatment & $-102 \times 10^{-6}$ & 0.024 \\
\hline The $A D C$ values of acute plaque after treatment & $-64 \times 10^{-6}$ & 0.140 \\
\hline The ADC values of the normal-appearing white matter before treatment & $-34.45 \times 10^{-6}$ & $<0.001$ \\
\hline The ADC values of normal-appearing white matter after treatment & $-14.55 \times 10^{-6}$ & 0.086 \\
\hline The ADC values of chronic plaque before treatment & $-505 \times 10^{-6}$ & $<0.001$ \\
\hline The $A D C$ values of chronic plaque after treatment & $-495 \times 10^{-6}$ & $<0.001$ \\
\hline
\end{tabular}

sponses detected in chronic plaques may be induced by irreversible CNS damages in this pathology. Increased ADC within NAWM can be explained by the inflammatory reaction and microscopic pathology (Caramia et al., 2002). Hence, detecting microscopic pathology in early phases in this region may promote recovery of NAWM pathology, inflammatory process, and delayed axonopathy if early and appropriate treatment is used.

We noticed a significant correlation between EDSS changes and mean ADC values changes of both acute and NAWM; however, a poor correlation was found between changes of EDSS count and ADC value changes in chronic plaques.

Axonal injury is a critical feature in MS lesions, occurring in the acute, chronic plaques and NAWM and strongly correlates with the patient's disability. In other words, this strong correlation could result from the inflammatory process and axonal injury recovery due to corticosteroid treatment. Additionally, these inflammatory changes lead to future neurodegenerative changes.

Thus, a strong correlation between changes in ADC values of acute plaques and NAWM and EDSS counts may be due to the effect of steroid therapies in accelerating inflammatory and axonal injury recovery. In addi- tion, these inflammatory changes have an essential role in the future clinical disability of patients.

Corticosteroids remain the principal therapy for patients with acute attack as it helps reduce clinical exacerbation rate, severity, and duration of symptom and inhibit diffuse microscopic changes in patients. In part of our study, there is an inconsistency between our result and Tuna Sahin's study (Sahin, Bozgeyik, Menzilcioglu, Citil, \& Erbay, 2015). Tuna Sahin et al. detected that mean ADC values in acute and chronic plaques decreased after therapy. But our results suggest a significant drop only in acute plaques, and these changes are not specific in the chronic plaques.

This study demonstrated increased ADC values in NAWM before treatment in patients with an acute attack which was per the results of the survey of Caramia et al. and Yurtsever et al. In addition, in the study of Yurtsever et al. like our study, ADC values of acute plaques were more than the control group (Caramia et al., 2002; Yurtsever et al., 2008). Furthermore, our results suggested an agreement with the study of Gondek et al. however. These two studies used a different kind of therapy for patients (Gondek, Zacharzewska-Gondek, Bladowska, Pokryszko-Dragan, \& Sasiadek, 2015).

Table 5. The relationship between variables and differences in EDSS values before and after treatment

\begin{tabular}{lc}
\hline \multicolumn{1}{c}{ Variables } & Correlations \\
\hline The difference in ADC values of acute plaque before and after treatment & 0.574 \\
\hline The difference in ADC values of chronic plaque before and after treatment & 0.272 \\
\hline The difference in ADC values of NAWM before and after treatment & 0.522 \\
\hline The difference in the number of enhancements before and after treatment & 0.246 \\
\hline
\end{tabular}


The number of enhancing plaques before and after treatment was counted in our study. The results suggested that the number of enhanced plaque significantly decreased after treatment. Our results align with the study of Miller et al. (1992) reporting a reduction of BloodBrain Barrier (BBB) abnormalities in $96 \%$ of enhancing lesions. Furthermore, we found a strong positive correlation between changes in EDSS count and changes in the number of improved plaques. Additionally, moderate and positive correlations were found between enhanced plaque count changes and mean ADC values of acute plaque.

Contrast enhancement reflects a breakdown of the blood-brain barrier, which correlates with lesions' inflammatory phase and activity (Balashov, Aung, DhibJalbut, \& Keller, 2011). For managing MS relapses, IV methylprednisolone directly affects the blood-brain barrier, thus resulting in lower gadolinium enhancement on magnetic resonance imaging. This process is thought to be mediated by suppressing inflammation, edema and restoring the blood-brain barrier, which accelerates recovery from attacks of MS (Martínez Cáceres, Barrau, Brieva, Espejo, Barbera, \& Montalban, 2002).

Therefore, the potential of MRI measurement in assessing and monitoring efficacy is increasingly being recognized and appreciated. With the approval of the new and more effective MS therapeutics, the spectrum of MRI techniques in treatment monitoring has become broader (Wattjes, Steenwijk, \& Stangel, 2015). Gd-enhancing $\mathrm{T}_{1} \mathrm{w}$ has poor sensitivity and specificity for the underlying pathophysiologic process (Zivadinov, Stosic, Cox, Ramasamy, \& Dwyer, 2008). Therefore, using advanced techniques such as DWI as a supplementary modality can help us predict neurologic dysfunction and evaluate treatment with high sensitivity.

\section{Conclusion}

This study indicated that the mean ADC for acute plaques and normal white matter decreased significantly after treatment. However, the mean ADC value in chronic plaques did not significantly change despite decreasing. In addition, there was a strong correlation between variations in EDSS, the mean ADC for acute plaques, and normal white matter. Variations in EDSS, ADC for chronic plaques revealed a weak relationship. This study also showed that the number of enhanced plaques decreased after treatment, and there was a positive correlation between its variations and EDSS variations.

\section{Ethical Considerations}

\section{Compliance with ethical guidelines}

This study was approved by the Research Council of Shahid Beheshti University of Medical Sciences (Code: IR.SBMU.RETECH.REC.1396.658).

\section{Funding}

This research did not receive any grant from funding agencies in the public, commercial, or non-profit sectors.

\section{Authors' contributions}

All authors equally contributed to preparing this article.

\section{Conflict of interest}

The authors declared no conflict of interest.

\section{Refrences}

Almolla, R. M., Hassan, H. A., Raya, Y. M., \& Hussein, R. A. (2016) Correlation of apparent diffusion coefficient to cognitive impairment in relapsing remittent multiple sclerosis (plaque, periplaque and normal appearing white matter). The Egyptian Journal of Radiology and Nuclear Medicine, 47(3), 1009-18. [DOI:10.1016/j. ejrnm.2016.04.018]

Aung, W. Y., Mar, S., \& Benzinger, T. L. (2013). Diffusion tensor MRI as a biomarker in axonal and myelin damage. Imaging in Medicine, 5(5), 427-40. [DOI:10.2217/iim.13.49] [PMID] [PMCID]

Balashov, K. E., Aung, L. L., Dhib Jalbut, S., \& Keller, I. A. (2011). Acute multiple sclerosis lesion: Conversion of restricted diffusion due to vasogenic edema. Journal of Neuroimaging, 21(2), 2024. [DOI:10.1111/j.1552-6569.2009.00443.x] [PMID] [PMCID]

Caramia, F., Pantano, P., Di Legge, S., Piattella, M. C., Lenzi, D., \& Paolillo, A., et al. (2002). A longitudinal study of MR diffusion changes in normal appearing white matter of patients with early multiple sclerosis. Magnetic Resonance Imaging, 20(5), 383-8. [DOI:10.1016/S0730-725X(02)00519-2] [PMID]

Davoudi, Y., Foroughipour, M., Torabi, R., Layegh, P., Matin, N., \& Shoeibi, A. (2016). Diffusion weighted imaging in acute attacks of multiple sclerosis. Iranian Journal of Radiology, 13(2), e21740. [DOI:10.5812/iranjradiol.21740] [PMID] [PMCID]

Eisele, P., Szabo, K., Griebe, M., Rossmanith, C., Förster, A., \& Hennerici, M., et al. (2012). Reduced diffusion in a subset of acute MS lesions: A serial multiparametric MRI study. American Journal of Neuroradiology, 33(7), 1369-73. [DOI:10.3174/ajnr.A2975] [PMID] [PMCID]

Etemadifar, M., Sajjadi, S., Nasr, Z., Firoozeei, T. S., Abtahi, S. H., \& Akbari, M., et al. (2013). Epidemiology of multiple sclerosis in Iran: A systematic review. European Neurology, 70(5-6), 356-63. [DOI:10.1159/000355140] [PMID] 
Ghasemi, N., Razavi, S., \& Nikzad, E. (2017). Multiple sclerosis: Pathogenesis, symptoms, diagnoses and cell-based therapy. Cell Journal, 19(1), 1-10. [DOI:10.22074/cellj.2016.4867] [PMID][PMCID]

Goldenberg, M. M. (2012). Multiple sclerosis review. Pharmacy and Therapeutics, 37(3), 175-84. [PMID][PMCID]

Gondek, T. M., Zacharzewska-Gondek, A., Bladowska, J., Pokryszko-Dragan, A., \& Sasiadek, M. (2015). P. 1. i. 020 Changes in apparent diffusion coefficient values in patients with multiple sclerosis with regard to treatment with interferon beta. European Neuropsychopharmacology, 25(2), S310-S11. [DOI:10.1016/S0924-977X(15)30379-5]

Hannoun, S., Roch, J. A., Durand Dubief, F., Vukusic, S., Sappey Marinier, D., \& Guttmann, C. R., et al. (2015). Weekly multimodal MRI follow-up of two multiple sclerosis active lesions presenting a transient decrease in ADC. Brain and Behavior, 5(2), e00307. [DOI:10.1002/brb3.307] [PMID] [PMCID]

Martínez Cáceres, E. M, Barrau, M. A, Brieva, L., Espejo, C., Barbera, N., \& Montalban, X. (2002). Treatment with methylprednisolone in relapses of multiple sclerosis patients: Immunological evidence of immediate and short-term but not long-lasting effects. Clinical \& Experimental Immunology, 127(1), 165-71. [DOI:10.1046/j.1365-2249.2002.01725.x] [PMID] [PMCID]

Miller, D. H., Thompson, A. J., Morrissey, S. P., MacManus, D. G., Moore, S. G., \& Kendall, B. E., et al. (1992). High dose steroids in acute relapses of multiple sclerosis: MRI evidence for a possible mechanism of therapeutic effect. Journal of Neurology, Neurosurgery \& Psychiatry, 55(6), 450-3. [DOI:10.1136/ jnnp.55.6.450] [PMID] [PMCID]

Sahin, T., Bozgeyik, Z., Menzilcioglu, M. S., Citil, S., \& Erbay, M. F. (2015). Importance of diffusion weighted magnetic resonance imaging in evaluation of the treatment efficacy in multiple sclerosis patients with acute attacks. Polish Journal of Radiology, 80, 544-8. [DOI:10.12659/PJR.895325] [PMID] [PMCID]

Simon, J. H. (1997). Contrast-enhanced MR imaging in the evaluation of treatment response and prediction of outcome in multiple sclerosis. Journal of Magnetic Resonance Imaging, 7(1), 29-37. [DOI:10.1002/jmri.1880070106] [PMID]

Wattjes, M. P., Steenwijk, M. D., \& Stangel, M. (2015). MRI in the diagnosis and monitoring of multiple sclerosis: An update. Clinical Neuroradiology, 25(2), 157-65. [DOI:10.1007/s00062015-0430-y] [PMID]

Yurtsever, I., Hakyemez, B., Taskapilioglu, O., Erdogan, C., Turan, O. F., \& Parlak, M. (2008). The contribution of diffusion-weighted MR imaging in multiple sclerosis during acute attack. European Journal of Radiology, 65(3), 421-6. [DOI:10.1016/j.ejrad.2007.05.002] [PMID]

Zivadinov, R., Stosic, M., Cox, J. L., Ramasamy, D. P., \& Dwyer, M. G. (2008). The place of conventional MRI and newly emerging MRI techniques in monitoring different aspects of treatment outcome. Journal of Neurology, 255(1), 61-74 [DOI:10.1007/s00415-008-1009-1] [PMID] 